Ahmed Y. Sayed / Engineering Research Journal, 172 (December, 2021/ PH1-PH18)

\title{
CAPILLARY GRAVITATIONAL STABILITY OF STREAMING FLUID JET PERVADED BY OBLIQUE VARYING MAGNETIC FIELD
}

\author{
Ahmed Y. Sayed \\ Engineering Physics and Mathematics Department, Faculty of Engineering (Mataria), Helwan \\ University, Cairo, Egypt. \\ Email: ahmed_badr@m-eng.helwan.edu.eg \\ ORCID: 0000-0001-5691-4993
}

\begin{abstract}
The stability of a streaming jet surrounded by self-gravitating tenuous medium and pervaded by oblique varying magnetic field under the combined effect of the capillary, selfgravitating and electromagnetic forces, has been discussed. The problem is formulated, the basic equations are solved upon applying appropriate boundary conditions, the dispersion relation is derived and discussed analytically and the results are verified numerically. The streaming has a strong destabilizing effect for all kinds of perturbation. For the axisymmetric mode $(m=0)$ the capillary force is destabilizing in the domain $0<x<1$, the self-gravitating force is destabilizing in the domain $0<x<1.0667$ and the electromagnetic force is destabilizing in all domains $0 \leq x<\infty$. For the non-axisymmetric mode, $m \geq 1$, both the capillary and self-gravitating forces are stabilizing while the electromagnetic force is stabilizing or destabilizing according to restriction. This means that for small values of $\beta$, the destabilizing action of the electromagnetic force could be neglected. Then the model under consideration will be purely stable in all modes for all, $m \geq 1$, short and long wavelengths.
\end{abstract}

Keywords: Oblique, magnetic, capillary, self-gravitating, fluid jet.

\section{INTRODUCTION}

The instability of a full fluid jet subjected to varying magnetic field, electric filed, selfgravitating forces and capillary force...etc., has been documented and mastered through numerous investigations [4] to [16]. That is due to its practical applications in several domains such as fuel atomization, spinning of synthetic fibers, the spray drying, ink jet printer ... etc.

The hydrodynamic instability of an ideal (or non-ideal) full fluid jet endowed with surface tension has been the concern of many investigators since the pioneering works of Rayleigh [30], Lin [29] and Drazin \& Reid [17]. Rayleigh (the 3rd Lord) derived the stability criterion and laid the mathematical foundation for analyzing such problems.

The stability analysis of an ideal fluid cylinder under the action of the capillary force reveals that the cutoff wavenumbers normalized with respect to the radius of the cylinder is unit, while the maximum growth rate of instability occurs as that wavenumbers about. Such kind of study has been carried out in the axisymmetric perturbation mode, for the first time, by Chandrasekhar and Fermi [11] on utilizing the technique of presenting the solenoidal vectors in terms of poloidal and toroidal quantities.

Soon afterwards Ogansian [28] studied that problem via the technique of normal mode analysis. Chandrasekhar [16] studied the stability of a full liquid jet subject to the capillary force and also, separately, under the self-gravitating forces for all axisymmetric and non-axisymmetric perturbation modes. Moreover, he [16] examined the effect of constant 
magnetic field on the capillary instability of full fluid jet in the axisymmetric mode by using the technique of presenting solenoidal vectors in terms of toroidal and poloidal quantities.

The same has been done by Chandrasekhar for a fluid cylinder under the action of the self-gravitating and Lorentz (with a uniform magnetic field) forces. In the last decades, more attention paid for elaborating more comprehensive MHD stability problems [31] - [36]. The stability of different cylindrical models under the action of self-gravitating force in addition to other forces has been elaborated by Radwan and Hasan [36] and [35]. Apart from the mathematical complexities arising in instability analysis with such disturbances as pointed out by Chandrasekhar [16] there is an overriding reason for studying the response of the system to these disturbances. In the absence of magnetic diffusion non-axisymmetric disturbances generally twist magnetic lines of force and produce an intimate coupling between the hydrodynamic and hydromagnetic influences that prevent the latter from being masked whatever is the smallest value of the magnetic field intensity. Axisymmetric disturbances, on the other hand, only bend the lines of force and under certain circumstances MHD effects may dominate over those due to magnetic forces.

Hasan [19] has investigated the stability of an oscillating streaming fluid cylinder subjected to the combined effect of the capillary, selfgravitating and electrodynamic forces in all axisymmetric and non-axisymmetric perturbation modes. He [20] has investigated the stability of oscillating streaming self-gravitating dielectric incompressible fluid cylinder surrounded by tenuous medium of negligible motion pervaded by transverse varying electric field for all the axisymmetric and non-axisymmetric perturbation modes. He [21] has studied the instability of a full fluid cylinder surrounded by self-gravitating tenuous medium pervaded by transverse varying electric field under the combined effect of the capillary, self-gravitating, and electric forces for all the modes of perturbations. He [22] the magnetodynamic stability of a fluid jet pervaded by transverse varying magnetic field while its surrounding tenuous medium is penetrated by uniform magnetic field.

Several attempts to determine the effects of compressibility on the linearized RayleighTaylor instabilities development have been made by a number of researchers [10]-[39], but the results were in disagreement with each other. In plasmas Rayleigh-Taylor instabilities has been studied for stratified incompressible layer in the presence of magnitude of the gravitational acceleration by Goldston and Rutherford [37]. RTI has been considered in inhomogeneous plasma rotating uniformly in an external magnetic field (vertical or horizontal-direction) by Al-Khateeb and Laham [8]-[7]. The interaction between plasma and a rotating magnetic field have been studied by several authors. For example, the theoretical works have been done by Moffat [23], Kono and Tanaka [26], Grants and Gerebeth [24]. Experimental investigations of this problem have been done by Volz and Mazuruk [27] and Nagaoka et al. [25]. Hasan and Abdelkhalek [9] has studied the magnetogravitodynamic stability of a streaming fluid cylinder and examining the influence of capillary and magnetic forces on the self-gravitating instability of the present models. This may be carried out, for all axisymmetric and non-axisymmetric modes of perturbation. Hasan [2] had studied the linear stability of self-gravitating compound dielectric immiscible jets under the influence of an axial electric field. The jets are streaming along the axis with alternating velocity .

Guided by this motivation here we study the instability of a full fluid cylinder surrounded by self-gravitating tenuous medium pervaded by oblique varying magnetic field under the combined effect of the capillary, self-gravitating and electromagnetic forces for all modes of perturbations. 


\section{FORMULATION OF THE PROBLEM}

Consider a self-gravitating fluid cylinder of radius $R_{o}$ surrounded by self-gravitating tenuous medium of negligible inertia. The fluid is assumed to be incompressible, inviscid, perfectly conducting and strcaming with velocity $\underline{u}_{0}=(0,0, U)$, where $U$ is the fluid velocity component in $z$ direction of the cylindrical coordinates $(r, \phi, z)$. The fluid cylinder pervaded by the uniform magnetic field

$$
\underline{H}_{\circ}=\left(0,0, H_{\circ}\right),
$$

and the surrounding self-gravitating tenuous medium is being penetrated by the oblique varying magnetic field

$$
\underline{H}_{o}^{l n}=\left(0, \beta H_{o} R_{o} r^{-1}, \alpha H_{o}\right),
$$

where $H_{o}$ is the intensity of the magnetic field in the fluid while $\alpha$ and $\beta$ are some parameters. The components of $\underline{H}_{0}$ and $\underline{H}_{o}^{t n}$ are considered along the utilizing cylindrical coordinates $(r, \varphi, z)$ system with $z$-axis coinciding with the axis of the fluid cylinder. The model is acting upon the combined effect of the self-gravitating and electromagnetic forces in addition to the capillary force along the perturbed fluid interface, see Figure (1).

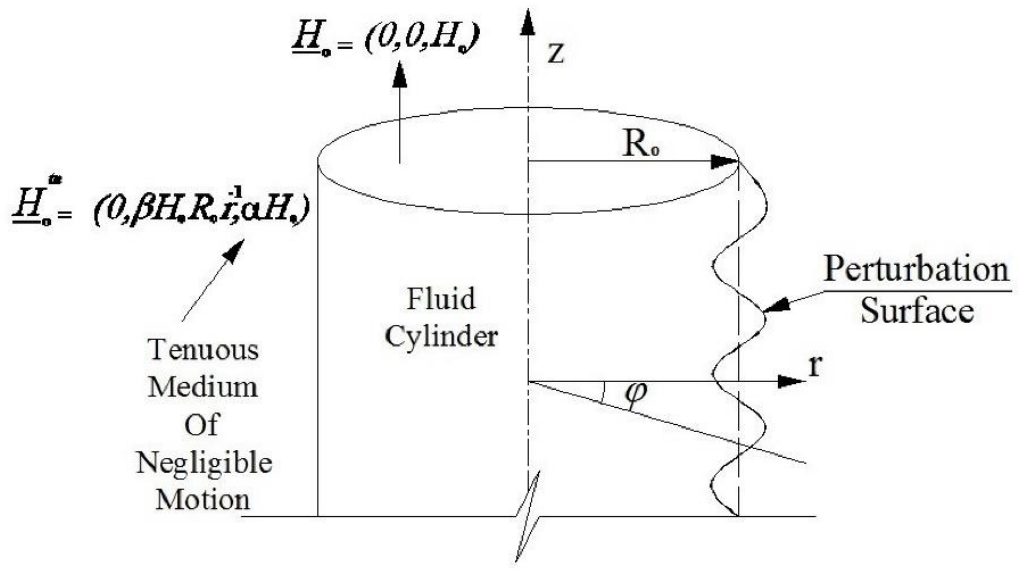

Figure 1: Sketch of MHD Fluid Jet.

The basic equations are the combinations of the ordinary hydrodynamic equations, Maxwell equations concerning the electromagnetic theory and Newtonian self-gravitating equations. Under the present circumstances, for the problem at hand, the fundamental equations are given as follows.

In the fluid

$$
\begin{aligned}
\rho\left(\frac{\partial \underline{u}}{\partial t}+(\underline{u} \cdot \nabla) \underline{u}\right) & =-\nabla P+\mu(\nabla \wedge \underline{H}) \wedge \underline{H}+\rho \nabla V, \\
\nabla \cdot \underline{H} & =0, \\
\nabla \cdot \underline{u} & =0, \\
\frac{\partial \underline{H}}{\partial t} & =\nabla \wedge(\underline{u} \wedge \underline{H})=(\underline{H} \cdot \nabla) \underline{u}-(\underline{u} \cdot \nabla) \underline{H}, \\
\nabla^{2} V & =-4 \pi \rho G .
\end{aligned}
$$


Along the fluid-tenuous medium

$$
P_{s}=T\left(\nabla \cdot \underline{N}_{s}\right)
$$

In the surrounding tenuous medium

$$
\begin{aligned}
\nabla \cdot \underline{H}^{t n} & =0 \\
\nabla \wedge \underline{H}^{t n} & =0,(\text { there is no current) } \\
\nabla^{2} V^{t n} & =0
\end{aligned}
$$

Here $\rho, \underline{u}$ and $P$ are the fluid density, velocity vector and kinetic pressure, $\mu$ and $\underline{H}$ are the magnetic permeability coefficient and magnetic field intensity and idem $\underline{H}^{t n}$. $V$ and $G$ are the self-gravitating potential and self-gravitating constant and idem $V^{t n}$. Equation (3) is the magnetogravitodynamic vector equation of motion involving the selfgravitating force $(\rho \nabla V)$, the pressure gradient force $(-\nabla P)$ and the electromagnetic force $\mu(\nabla \wedge \underline{H}) \wedge \underline{H}=\mu(\underline{H} \cdot \underline{\nabla}) \underline{H}-(\mu / 2) \nabla(\underline{H} \cdot \underline{H})$. Equations (4) and (9) are the conservation of magnetic flux which are identically satisfied. Equation (5) is the continuity equation appropriate for an incompressible fluid. Equation (6) is the evolution equation of magnetic field derived from Maxwell equations concerning the electromagnetic theory and the same for Equation (10) where there is no current in the tenuous medium. Equation (7) is the Newtonian self-gravitating equation of the fluid, and the same for Equation (11) where the tenuous medium is being of negligible motion. Equation (8) is being the equation of the capillary force, where $P_{s}$ is the surface pressure due to the curvature of the perturbed fluidtenuous medium, $T$ is the surface tension coefficient and $\underline{N}=\nabla f(r, \varphi, z) /|\nabla f(r, \varphi, z)|$ is a unit outward vector normal to the fluid-tenuous medium where $f(r, \varphi, z)=0$ is the equation of the boundary surface.

The system of basic Equations (3) - (11) are solved in the unperturbed state with $\underline{u}_{0}=(0,0, U)$ and cylindrical symmetries $\frac{\partial}{\partial \varphi}=0$ and $\frac{\partial}{\partial z}=0$. The appropriate boundary conditions are applied at the fluid-tenuous medium interface $r=R_{o}$ for determining the integration constants, and apart from the singular solutions we have obtained the following results:

$$
\begin{aligned}
V_{o} & =-\pi G \rho r^{2}, \\
V_{o}^{t n} & =2 \pi G \rho R_{o}^{2}\left[\ln \left(\frac{R_{o}}{r}\right)-\frac{1}{2}\right], \\
P_{o s} & =\frac{T}{R_{o}} .
\end{aligned}
$$

Upon applying the balance of the pressure across the fluid-tenuous medium interface at $r=R_{o}$, the distribution of the unperturbed fluid kinetic pressure is given by

$$
P_{o}=\frac{T}{R_{o}}+\pi G \rho^{2}\left(R_{o}^{2}-r^{2}\right)+\frac{\mu H_{o}^{2}}{2}\left(\alpha^{2}+\beta^{2}-1\right) .
$$

The first term in the right side of Equation (15) is the contribution due to the capillary force, the second one is due to the self-gravitating force while the third term is the magnetodynamic pressure due to the acting of the electromagnetic forces interior and exterior the fluid cylinder. At $r=R_{o}$, the self-gravitating force has no contribution. Moreover, if, $\alpha^{2}+\beta^{2}=1$, this means that the pervading magnetic fields inside and outside the fluid cylinder are the same, we have $P_{o}=\frac{T}{R_{o}}>0$. Therefore $P_{o}$ always has a value in the unperturbed state and this is essential for existing the model (it will not collapse). 


\section{PERTURBATION STATE}

For small departures from the unperturbed state, every variable quantity $Q(r, \varphi, z ; t)$ could be expanded as

$$
Q(r, \varphi, z ; t)=Q_{o}(r)+\varepsilon(t) Q_{1}(r, \varphi, z)+\ldots,
$$

where the suffices 0 and 1 denote the quantities in the unperturbed and fluctuating parts, respectively. Here $Q(r, \varphi, z ; t)$ stands for $\underline{u}, P, \underline{H}, \underline{H}^{t n}, V, V^{t n}, \underline{N}_{s}$ and the radial perturbed distance of the fluid cylinder. $\varepsilon(t)$ is non-dimensionless parameter providing a measure of the size of the disturbance. In reality $\varepsilon(t)$ is the amplitude of the perturbation at an instant time $t$, and we will specify it to be of the form

$$
\varepsilon(t)=\varepsilon_{o}(t) \exp (\sigma t),
$$

with $\varepsilon_{o}$, the value of $\varepsilon$ at $t=0$, is the initial amplitude and $\sigma$ is the growth rate or rather, if $\sigma=i \omega$ is imaginary, the oscillation frequency concerning the stability states. Since we are considering a departure from an unperturbed right-cylindrical shape of an incompressible fluid, a normal mode can be expressed uniquely in terms of the perturbed fluid-tenuous medium interface. Suppose, then that the perturbed interface is described by equation

$$
r=R_{o}+\eta(\varphi, z, t), \quad \eta \ll R_{o} .
$$

With

$$
\eta=\varepsilon(t) \exp (i(k z+m \varphi)),
$$

where $k$, any real number, is the longitudinal wave number along $z$-direction while $m$, an integer, is the azimuthal wave number. Here $\eta(\varphi, z, t)$ is the elevation of the free fluid-tenuous medium interface measured from the unperturbed position.

By utilizing the expansion (16), the basic equations (3) - (11) describing the motion of the fluid and the surrounding tenuous medium are linearized and then the relevant perturbation equations are given as follows.

In the fluid

$$
\begin{aligned}
\frac{\partial \underline{u}_{1}}{\partial t}+\left(\underline{u}_{0} \cdot \nabla\right) \underline{u}_{1}-\frac{\mu}{2 \rho}\left(\underline{H}_{0} \cdot \nabla\right) \underline{H}_{1} & =-\nabla \Pi_{1}, \\
\left(\frac{P_{1}}{\rho}\right)+\frac{\mu}{2 \rho}\left(\underline{H}_{0} \cdot \underline{H}_{1}\right)-V_{1} & =\Pi_{1}, \\
\nabla \cdot \underline{u}_{1} & =0, \\
\nabla \cdot \underline{H}_{1} & =0, \\
\left(\underline{H}_{0} \cdot \nabla\right) \underline{u}_{1}-\left(\underline{u}_{1} \cdot \nabla\right) \underline{H}_{0} & =\frac{\partial \underline{H}_{1}}{\partial t}, \\
\nabla^{2} V_{1} & =0 .
\end{aligned}
$$

Along the fluid-tenuous medium interface

$$
P_{1 s}=-\frac{T}{R_{o}^{2}}\left(\eta+\frac{\partial^{2} \eta}{\partial \varphi^{2}}+R_{o}^{2} \frac{\partial^{2} \eta}{\partial z^{2}}\right)
$$

In the tenuous medium surrounding the fluid

$$
\begin{aligned}
\nabla \cdot \underline{H}_{1}^{t n} & =0 \\
\nabla \wedge \underline{H}^{t n} & =0, \\
\nabla^{2} V_{1}^{t n} & =0 .
\end{aligned}
$$

where $P_{1}+(\mu / 2)\left(\underline{H}_{0} \cdot \underline{H}_{1}\right)=\rho\left(\Pi_{1}+V_{1}\right)$ is the total magneto-hydrodynamic pressure, which is the sum of the kinetic and magnetic pressures. 
Applying the divergence operator on the Equation (20) and using Equations (22) and (23), we get

$$
\nabla^{2} \Pi_{1}=0
$$

Equation (28) means that the relevant perturbed magnetic field, may be derived from a scalar function, viz.,

$$
\underline{H}_{1}^{t n}=-\nabla^{2} \Psi_{1}^{t n} .
$$

If we combine Equations (31) and (27), we obtain

$$
\nabla^{2} \Psi_{1}^{t n}=0 .
$$

Since we seek a solution whose $t$-space dependence is given by $\exp (i(k z+m \varphi)+\sigma t)$ times an amplitude function of $r$. Based on the linear perturbation technique that is being used for stability analysis, every perturbed quantity $Q_{1}(r, \varphi, z, t)$ may be expressed as

$$
Q_{1}(r, \varphi, z, t)=Q_{1}(r) \exp (i(k z+m \varphi)+\sigma t) .
$$

So the linear perturbation Equations (20) - (29) could be solved upon solving Laplace equations (25), (29), (30) and (32). Taking into account that the other variables $\underline{H}_{1}$ and $\underline{u}_{1}$ could be identified from

$$
\underline{H}_{1}=\frac{i k H_{o}}{(\sigma+i k U)} \underline{u}_{1}
$$

and

$$
\underline{u}_{1}=\frac{-(\sigma+i k U)}{(\sigma+i k U)^{2}+\Omega_{A}^{2}} \nabla \Pi_{1}
$$

where

$$
\Omega_{A}=\left(\frac{\mu H_{o}^{2} k^{2}}{\rho}\right)^{\frac{1}{2}}
$$

is the Alfven wave frequency.

By the use of the expansion (33) for equations (29), (30) and (32) we have

$$
r^{-1} \frac{d}{d r}\left(r \frac{d Q_{1}(r)}{d r}\right)-\left(\frac{m^{2}}{r^{2}}+k^{2}\right) Q_{1}(r)=0
$$

where $Q_{1}(r)$ stands for $\Pi_{1}(r), V_{1}(r), V_{1}^{t n}(r)$ and $\Psi_{1}^{t n}(r)$. The solution of the ordinary second order differential Equation (37) is given in terms of the ordinary Bessel functions with imaginary arguments of order $m$. Apart from singular solution, we have

$$
\begin{aligned}
V_{1} & =A I_{m}(k r) \exp (i(k z+m \varphi)+\sigma t), \\
V_{1}^{t n} & =A^{t n} I_{m}(k r) \exp (i(k z+m \varphi)+\sigma t), \\
\Pi_{1} & =B I_{m}(k r) \exp (i(k z+m \varphi)+\sigma t), \\
\Psi_{1}^{t n} & =C K_{m}(k r) \exp (i(k z+m \varphi)+\sigma t),
\end{aligned}
$$

where $I_{m}(k r)$ and $K_{m}(k r)$ are the modified Bessel functions of the first and second kind of order $m$, while $A, A^{t n}$, and $C$ are constants of integration to be determined.

\section{STABILITY CRITERION}

The solution of the basic Equations (3) - (11) in the unperturbed state given by equations (12) - (15) and in the perturbed state given by (34), (35) and (38) - (41) must satisfy certain boundary conditions across the fluid-tenuous medium interface at $r=R_{0}$. These appropriate boundary conditions could be applied as follows. 


\subsection{KINEMATIC CONDITION}

The normal component of the velocity vector must be compatible with the velocity of the fluid-tenuous medium interface at $r=R_{o}$. This condition is being

$$
\underline{N} \cdot \underline{u}=\frac{\partial r}{\partial t}
$$

where

$$
\underline{N}=(1,0,0)+\varepsilon_{o}\left(0, \frac{i m}{R_{0}},-i k\right) \exp (i(k z+m \varphi)+\sigma t) .
$$

From which, we get

$$
B=\frac{-R}{x \dot{I}_{m}(x)}\left((\sigma+i k U)^{2}+\Omega_{A}^{2}\right),
$$

where $x=k R_{o}$ is, dimensionless, the longitudinal wave number.

\subsection{SELF-GRAVITATING CONDITION}

The self-gravitating potential $V=V_{o}+\varepsilon V_{1}+\ldots$ and its derivative must be continuous across the perturbed fluid-tenuous medium interface (18) at $r=R_{o}$. These conditions are given as:

$$
\begin{aligned}
V_{1}+\eta \frac{\partial V_{o}}{\partial r} & =V_{1}^{t n}+\eta \frac{\partial V_{o}^{t n}}{\partial r} \\
\frac{\partial V_{1}}{\partial r}+\eta \frac{\partial^{2} V_{o}}{\partial r^{2}} & =\frac{\partial V_{1}^{t n}}{\partial r}+\eta \frac{\partial^{2} V_{o}^{t n}}{\partial r^{2}}
\end{aligned}
$$

From which, we get.

$$
\begin{aligned}
A & =4 \pi G \rho R_{o} K_{m}(x), \\
A^{t n} & =4 \pi G \rho R_{o} I_{m}(x) .
\end{aligned}
$$

\subsection{MAGNETODYNAMIC CONDITION}

The normal component of the magnetic field $\underline{H}=\underline{H}_{o}+\varepsilon \underline{H}_{1}+\ldots$ must be also continuous across the fluid-tenuous medium interface (18) at the boundary $r=R_{o}$, i.e.,

$$
C=\frac{i H_{o}(m \beta+\alpha x)}{x \dot{K}_{m}(x)} .
$$

From the foregoing analysis, we see that all the variables of the problem in the perturbed and unperturbed states are completely identified. Here for the aim of stability analysis we have to go one step more to apply some compatibility condition. The present model is acted upon the combined effect of the capillary, self-gravitating and electromagnetic forces. The normal component of the total stresses must be continuous across the fluid-tenuous medium interface (18) at the boundary $r=R_{o}$,

This condition is being

$$
P_{1}+\eta \frac{\partial P_{o}}{\partial r}+\frac{\mu}{2}\left[\left(\left(\underline{H}_{o} \cdot \underline{H}_{1}\right)+\eta \frac{\partial\left(\underline{H}_{o} \cdot \underline{H}_{o}\right)}{\partial r}\right)-\left(\left(\underline{H}_{o} \cdot \underline{H}_{1}\right)^{t n}+\eta \frac{\partial\left(\underline{H}_{o} \cdot \underline{H}_{o}\right)^{t n}}{\partial r}\right)\right]=P_{1 s} .
$$


By substituting from the obtained results above about $P_{1}, \underline{H}_{1}, \underline{H}_{1}^{t n}, P_{1 s}, \eta, P_{o}, \underline{H}_{o}$ and $\underline{H}_{o}^{t n}$ in the condition (50), the following stability criterion is established:

$$
(\sigma+i k U)^{2}=\sigma_{T}^{2}+\sigma_{G}^{2}+\sigma_{\underline{H}_{o}}^{2}
$$

where

$$
\begin{aligned}
\sigma_{T}^{2} & =\frac{T}{\rho R_{o}^{3}}\left(1-m^{2}-x^{2}\right) F_{m}(x), \\
\sigma_{G}^{2} & =4 \pi G \rho\left(I_{m}(x) K_{m}(x)-\frac{1}{2}\right) F_{m}(x), \\
\sigma_{\underline{H}_{o}}^{2} & =\left(\frac{\mu H_{o}^{2}}{\rho R_{o}^{2}}\right)\left\{-x^{2}+\left[\beta^{2}+(\beta m+\alpha x)^{2}\left(G_{m}(x)\right)^{-1}\right] F_{m}(x)\right\},
\end{aligned}
$$

with

$$
G_{m}(x)=\frac{x \dot{K}_{m}(x)}{K_{m}(x)}, \quad \text { and, } \quad F_{m}(x)=\frac{x \dot{I}_{m}(x)}{I_{m}(x)}
$$

\section{LIMITING CASES}

The dispersion relations (51) is a relationship correlating the temporal amplification $\sigma$ or rather the oscillation frequency $\omega$ with the wavenumbers $x$ and $m$, the modified Bessel functions $I_{m}(x), K_{m}(x)$ and their derivation, the oblique magnetic field parameter $\alpha$ and $\beta$, the parameters $\rho, \underline{H}_{o}, G, T$ and $R_{o}$ of the problem and with the fundamental. Quantity $T /\left(\rho R_{o}^{3}\right)^{-1 / 2}$ as well as $(4 \pi G \rho)^{-1 / 2}$, and $\left(\rho R_{o}^{2} / \mu H_{o}^{2}\right)^{-1 / 2}$ as a unit of time.

Since the present problem is somewhat more general than the other studied problem, stability criteria of numerous problems of different character may be obtained from the general dispersion relation (51) as limiting cases with appropriate choices.

A lot of simplifications, like $U=0, G=0, \underline{H}_{o}=0$ and $m=0$, are essential to get the following dispersion relation from (51)

$$
\sigma^{2}=\frac{T}{\rho R_{o}^{3}} \frac{x \dot{I}_{0}(x)}{I_{0}(x)}\left(1-x^{2}\right),
$$

which is obtained by Rayleigh [30] for a naïve model. However, for $G=0, \underline{H}_{0}=0$ and $m \geq 1$, the relation (51) reduces to

$$
\sigma^{2}=\frac{T}{\rho R_{o}^{3}}\left(\frac{x \dot{I}_{m}(x)}{I_{m}(x)}\right)\left(1-m^{2}-x^{2}\right),
$$

which is derived and studied by Chandrasekhar [16].

If we suppose that $U=0, T=0, \underline{H}_{o}=0$ and $m=0$ the relation (51) degenerates to

$$
\sigma^{2}=4 \pi G \rho\left(\frac{x I_{o}(x)}{I_{o}(x)}\right)\left(I_{o}(x) K_{o}(x)-\frac{1}{2}\right),
$$

that coincides with the dispersion relation derived, for first time, by Chandrasekhar and Fermi [11].

However if $U=0, T=0, \underline{H}_{o}=0$ and $m \geq 1$, the relation (51) will be

$$
\sigma^{2}=4 \pi G \rho\left(\frac{x \dot{I}_{m}(x)}{I_{m}(x)}\right)\left(I_{m}(x) K_{m}(x)-\frac{1}{2}\right),
$$

which is derived and discussed by Chandrasekhar [16]. 
If $U=0, G=0, T=0$ and $\alpha=0$ while $m \geq 0$, the relation (51) yields

$$
\sigma^{2}=\left(\frac{\mu H_{o}^{2}}{\rho R_{o}^{3}}\right)\left\{-x^{2}+\left[\beta^{2}\left(1+m^{2}\right)\left(G_{m}(x)\right)^{-1}\right] F_{m}(x)\right\},
$$

which is the magnetodynamic dispersion relation of perfectly conducting fluid cylinder surrounded by transverse varying magnetic field, valid for all axisymmetric mode $m=0$ and non-axisymmetric modes $m \geq 1$, which is derived and discussed by Ahmed $\underline{\text { et }} \underline{\text { al. }}$. [38].

\section{STABILITY DISCUSSIONS}

In order to discuss the stability of the present model under the combined effect of the capillary, self-gravitational and electromagnetic forces, it is found more convenient that we should study some behavior of the modified Bessel functions appearing in the criterion (51).

For non-zero real value of $x$, the recurrence relations of the modified Bessel functions, see [1], are given in the form

$$
\begin{aligned}
2 \dot{I}_{m}(x) & =I_{m-1}(x)+I_{m+1}(x), \\
2 \dot{K}_{m}(x) & =-K_{m-1}(x)-K_{m+1}(x)
\end{aligned}
$$

Since for each non-zero real value of $x$ that $I_{m}(x)$ always positive and monotonic increasing while $K_{m}(x)$ is monotonic decreasing but never negative, we see that

$$
\dot{I}_{m}(x)>0 \text { and } \dot{K}_{m}(x)<0 .
$$

Therefore, for each non-zero real value of $x$, we have

$$
F_{m}(x)>0 \text { while } G_{m}(x)<0,
$$

in all axisymmetric mode $m=0$ and non-axisymmetric modes $m \geq 1$.

\subsection{CAPILLARY STABILITY}

The dispersion relation of the present model under the action of the capillary force is given by Equation (52).

By an appeal to the recurrence relation (61) and the inequalities (63) and (64), the discussion of the dispersion relation (52) reveals that the behavior of the non-dimension growth rate $\sigma /\left(\rho R_{o}^{3} / T\right)^{1 / 2}$ is mainly depend on the quantity $\left(1-m^{2}-x^{2}\right)$ since the associated function $F_{m}(x)$ is positive for each non-zero real values of $x$. We see that:

i. $\sigma^{2}<0$ as $m \geq 1$ for all values of $x$ in the range $0<x<\infty$,

ii. $\sigma^{2}<0$ as $m=0$ in the domain $1 \leq x<\infty$ where the equality corresponds to the marginal stability state,

iii. $\sigma^{2}>0$ as $m=0$ in the domain $0<x<1$.

This means that the main stability domains are $1<x<\infty$ as $m=0$ and $0<x<\infty$ as $m \geq 1$. While the only unstable domain is $0<x<1$ as $m=0$. The marginal stability state which is a transition from stability to instability states is given as $\sigma=0$ which occurs at $x=1$ for $m=0$. 
We conclude that the fluid cylinder is capillary stable to all non-axisymmetric modes $m \geq 1$ and also to axisymmetric (sausage) mode $m=0$ for perturbation whose wavelength $\lambda=2 \pi / k$ is shorter than the circumference $2 \pi R_{o}$ of the fluid cylinder. While it is unstable to sausage mode whose $\lambda$ is longer than $2 \pi R_{o}$. Clearly, if $\lambda=2 \pi R_{o}$ in the sausage mode $m=0$, we get neutral stability.

\subsection{SELF-GRAVITATING STABILITY}

The dispersion relation of a self-gravitating fluid cylinder surrounded by self-gravitating tenuous medium is given by Equation (53). Based on the relations (61), (62), the inequalities (63) and (64) concerning Bessel functions, the relation (53) is discussed and we found the following.

The determining of the sign of $\left(\sigma^{2} / 4 \pi G \rho\right)$ could be determined if the sign of the quantity $Q_{m}(x)=\left(I_{m}(x) K_{m}(x)-\frac{1}{2}\right)$ is known for different values of $m$ and $x$.

In the non-axisymmetric modes $m \geq 1$, it is found that $Q_{m}(x)$ is negative for all nonzero values of $x$. This means that $\sigma$ will be imaginary and consequently the fluid cylinder will be stable in $m \geq 1$ modes for all short and long wavelengths. In the axisymmetric mode $m=0$, it is found that the quantity $Q_{m}(x)$ may be positive or negative depending on the non-zero values of $x$. This means that the fluid cylinder may be unstable or stable in some states. Numerical and analytical studies of the relation (53) for $m=0$ show that the fluid cylinder is gravitational unstable in the domain $0<x<1.0667$ while it occurs stable in the domains $1.0667<x<\infty$ and the transition from the oscillation state to that of instability occurred at $x=1.0667$.

We conclude that the self-gravitating fluid cylinder surrounded by self-gravitating tenuous medium is unstable only in the range $0<x<1.0667$ as $m=0$ while it is stable in all other axisymmetric and non-axisymmetric states.

\subsection{MAGNETODYNAMIC STABILITY}

The absence of the combined effect of the capillary and self-gravitating forces and assuming that the fluid cylinder is acted by the electromagnetic forces in addition to the pressure gradient force, the dispersion relation of this case is given by Equation (54).

The influence of the axial magnetic field pervaded into the fluid cylinder is represented by the term $\left(-x^{2}\right)$ following the quantity $\left(\mu H_{o}^{2} / \rho R_{o}^{2}\right)$. It has always a stabilizing influence and that character is valid whether the perturbation is axisymmetric or nonaxisymmetric. The influence of the oblique magnetic field pervaded into the tenuous medium is represented by $\left[\beta^{2}+(\beta m+\alpha x)^{2}\left(G_{m}(x)\right)^{-1}\right] F_{m}(x)$ following the quantity $\left(\mu H_{o}^{2} / \rho R_{o}^{2}\right)$. Taking into account the inequality (64) we see that the term $\beta^{2} F_{m}(x)$ made the electromagnetic force destabilizing not only in $m=0$ mode, but also for $m \geq 1$ modes while the term $(\beta m+\alpha x)^{2}\left(G_{m}(x)\right)^{-1} F_{m}(x)$ shows that the electromagnetic force is stabilizing in the non-axisymmetric modes $m \geq 1$ but it has no effect at all in the axisymmetric mode $m=0$.

This means that for $m=0$ mode, in general, the electromagnetic force is purely destabilizing while for $m \geq 1$ modes it is stabilizing or destabilizing according to restrictions.

\subsection{HYDROMAGNETIC GRAVITATING STABILITY}

In this general case the self-gravitating fluid cylinder surrounded by tenuous selfgravitating tenuous medium of negligible motion is acted by the combined effect of the 
capillary, self-gravitating and electromagnetic forces. The dispersion relation of this general case is given by Equation (51). The discussion of this relation leads to the following.

i. The streaming has a strong destabilizing effect for all kinds of perturbation.

ii For $m=0$ mode: the capillary force is destabilizing in the domain $0<x<1$, the selfgravitating force is destabilizing in the domain $0<x<1.0667$. The electromagnetic force is destabilizing in all domains $0 \leq x<\infty$. This means that the model under consideration is purely unstable in the axisymmetric perturbation for all short and long wavelengths.

iii. For $m \geq 1$ mode: both the capillary and self-gravitating forces are stabilizing while the electromagnetic force is stabilizing or destabilizing according to restriction. This means that for small values of $\alpha$ and $\beta$, the destabilizing action of the electromagnetic force could be neglected. Then the model under consideration will be purely stable in all modes for all $m \geq 1$ short and long wavelengths.

Physically, the stabilizing effect of the exterior magnetic field is expected because it has been assumed that the pervading magnetic field is uniform.

Moreover, the stabilizing effect of the oblique magnetic field in the fluid region is due to the influence of Lorentz force that comes out of the interaction of the magnetic induction and the electric current produced due to the pervading magnetic field. Indeed, such electromagnetic force causes the following stresses [3].

The magnetic pressure $\frac{\mu}{2}(\underline{H} \cdot \underline{H})$ per unit area acting in all directions of the fluid (resistivity is neglected here) and an equal magnetic tension $\frac{\mu}{2}(\underline{H} \cdot \underline{H})$ per unit area acting along the magnetic lines of force. Due to these stresses the lines of force are able to endow the fluid with a sort of rigidity. The magnetic fields exert strong influence not only to the axisymmetric mode $(m=0)$ that causes only the bending of the magnetic lines of force, but also to non axisymmetric modes that lead to twisting of the lines of force.

\section{NUMERICAL ANALYSIS}

The numerical analysis has been carried out in order to identify and examine the magnetic field influence and surface tension and also the effect of the streaming and the self-gravitating force on the stability of the model. In addition to that the oscillation states and the transition points from these states to those of instability may be also determined for given values of the magnetic field intensity for $m=0$.

This has been elaborated by computing the dispersion relation

$$
\begin{aligned}
\sigma^{* 2}= & \left(1-x^{2}\right) F_{o}(x)+N\left(I_{o}(x) K_{o}(x)-\frac{1}{2}\right) F_{o}(x) \\
& +M\left(-x^{2}+\left[\beta^{2}+\alpha^{2} x^{2} \frac{K_{o}(x)}{K_{o}^{\prime}(x)}\right] F_{o}(x)\right)+U^{*},
\end{aligned}
$$

where

$\sigma^{*}=\sigma / \sqrt{T / \rho R_{o}^{3}}, N=\left(4 \pi G \rho^{2} R_{o}^{2}\right) / T$ and $M=H_{o}^{2} /\left(T / \mu R_{o}\right)$ are dimensionless quantities.

The dispersion relation (65) has been computed, by using the MATLAB program, for all short and long wavelengths $0 \leq x \leq 3$. The values of $\sigma^{* 2}$ corresponding to the unstable 


\section{Ahmed Y. Sayed / Engineering Research Journal, 172 (December, 2021/ PH1-PH18)}

domains and those of $\omega^{*}=\omega / \sqrt{T / \rho R_{o}^{3}}$ corresponding to the stable domains are collected and represented graphically. Such calculations have been elaborated for different values of $U, \alpha, \beta, M$ and $N$ for regular values of $x$ in the range $0 \leq x \leq 3$.

The numerical data are plotted graphically, see Figures (2)-(8). There are many features of interest in these numerical illustrations, we deduce the following:

1. For $(M, N)=(0.1 ; 0.1), U=0, \alpha=\beta=0.1,0.3,0.5,0.7$ and 1.0, see Figure (2): It is found that the unstable domains are $0<x<0.85321,0<x<0.86561$, $0<x<0.87531,0<x<0.88561$ and $0<x<0.92501$ respectively, while the stable domains are $0.85321 \leq x<\infty, 0.86561 \leq x<\infty, 0.87531 \leq x<\infty$, $0.88561 \leq x<\infty$ and $0.92501 \leq x<\infty$ respectively, where the equalities are associated with the marginal stability states.

2. For $(M, N)=(0.1 ; 0.1), U=0.5, \alpha=\beta=0.1,0.3,0.5,0.7$ and 1.0, see Figure (3): It is found that the unstable domains are $0<x<2.52501,0<x<2.55124$, $0<x<2.57541,0<x<2.85312$ and $0<x<2.95224$ respectively, while the stable domains are $2.52501 \leq x<\infty, 2.55124 \leq x<\infty, 2.57541 \leq x<\infty$, $2.85312 \leq x<\infty$ and $2.95224 \leq x<\infty$ respectively, where the equalities are associated with the marginal stability states.

3. For $(M, N)=(0.1 ; 0.5), U=0.5, \alpha=\beta=0.1,0.3,0.5,0.7$ and 1.0, see Figure (4): It is found that the unstable domains are $0<x<2.45351,0<x<2.47521$, $0<x<2.55640,0<x<2.52561$ and $0<x<2.58357$ respectively, while the stable domains are $2.45351 \leq x<\infty, 2.47521 \leq x<\infty, 2.55640 \leq x<\infty$, $2.52561 \leq x<\infty$ and $2.58357 \leq x<\infty$ respectively, where the equalities are associated with the marginal stability states.

4. For $(M, N)=(0.1 ; 1.0), U=0.5, \alpha=\beta=0.1,0.3,0.5,0.7$ and 1.0, see Figure (5): It is found that the unstable domains are $0<x<1.52513,0<x<1.55014$, $0<x<1.75431,0<x<1.91025$ and $0<x<2.48201$ respectively, while the stable domains are $1.52513 \leq x<\infty, 1.55014 \leq x<\infty, 1.75431 \leq x<\infty$, $1.91025 \leq x<\infty$ and $2.48201 \leq x<\infty$ respectively, where the equalities are associated with the marginal stability states.

5. For $(M, N)=(0.5 ; 0.5), U=0.5, \alpha=\beta=0.1,0.3,0.5,0.7$ and 1.0, see Figure (6): It is found that the unstable domains are $0<x<1.52513,0<x<1.55014$, $0<x<1.75431,0<x<1.91025$ and $0<x<2.48201$ respectively, while the stable domains are $1.52513 \leq x<\infty, 1.55014 \leq x<\infty, 1.75431 \leq x<\infty$, $1.91025 \leq x<\infty$ and $2.48201 \leq x<\infty$ respectively, where the equalities are associated with the marginal stability states.

6. For $(M, N)=(0.1 ; 0.1), U=0.5, \alpha=0,0.5,1.0,1.5$ and $2.0, \beta=0.1$, see Figure (7): It is found that the unstable domains are $0<x<2.52512,0<x<2.55321$, $0<x<2.57517,0<x<2.62587$ and $0<x<2.72123$ respectively, while the stable domains are $2.52512 \leq x<\infty, 2.55321 \leq x<\infty, 2.57517 \leq x<\infty$, $2.62587 \leq x<\infty$ and $2.72123 \leq x<\infty$ respectively, where the equalities are associated with the marginal stability states.

7. For $(M, N)=(0.1 ; 0.1), U=0.5, \alpha=0.1, \beta=0,0.5,1.0,1.5$ and 2.0, see Figure (8): It is found that the unstable domains are $0<x<2.52501,0<x<2.55365$, $0<x<2.57326,0<x<2.62577$ and $0<x<2.75012$ respectively, while the stable domains are $2.52501 \leq x<\infty, 2.55365 \leq x<\infty, 2.57326 \leq x<\infty$, 
$2.62577 \leq x<\infty$ and $2.75012 \leq x<\infty$ respectively, where the equalities are associated with the marginal stability states.

\section{CONCLUSION}

From the foregoing discussions and numerical analysis at $m=0$, we conclude the following :

1. For Figure (2) and Figure (3) with the same values of $M, N, \alpha, \beta$ but with $U=$ $0, U=0.5$ respectively. it is found that the unstable domains are increasing with increasing of $U$ values. This means that the streaming has a destabilizing effect on the model.

2. For Figure (4) and Figure (5) with the same values of $M, U, \alpha, \beta$ but with $N=$ $0.5, N=1.0$ respectively. it is found that the unstable domains are increasing with increasing of $N$ values. This means that the self-gravitating force has a destabilizing effect on the model.

3. For Figure (4) and Figure (6) with the same values of $N, U, \alpha, \beta$ but with $M=$ $0.1, M=0.5$ respectively. it is found that the unstable domains are increasing with increasing of $M$ values. This means that the electromagnetic force has a destabilizing effect on the model.

4. For Figure (7) and Figure (8) with the same values of $M, N, U$ but with different values $\alpha, \beta$ for respcetively. it is found that the unstable domains are increasing with increasing of $\alpha, \beta$ values. This means that the component of the electromagnetic force in $y$ direction alone or $z$ direction alone has a destabilizing effect on the model.

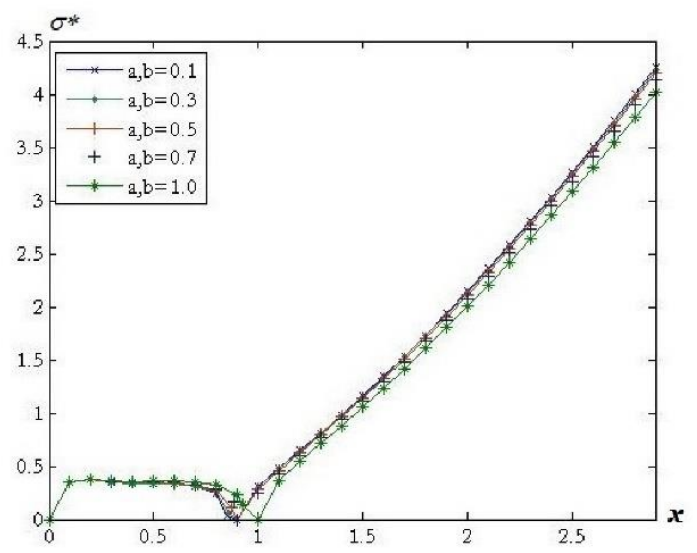

Figure 2: Stable and unstable domains of MHD gravitating jet penetrated by oblique varying magnetic field for $(M, N)=(0.1,0.1)$ with $\sigma^{*}=\sigma / \sqrt{T / \rho R_{o}^{3}}, U=0$ and $a=\alpha, B=\beta$. 


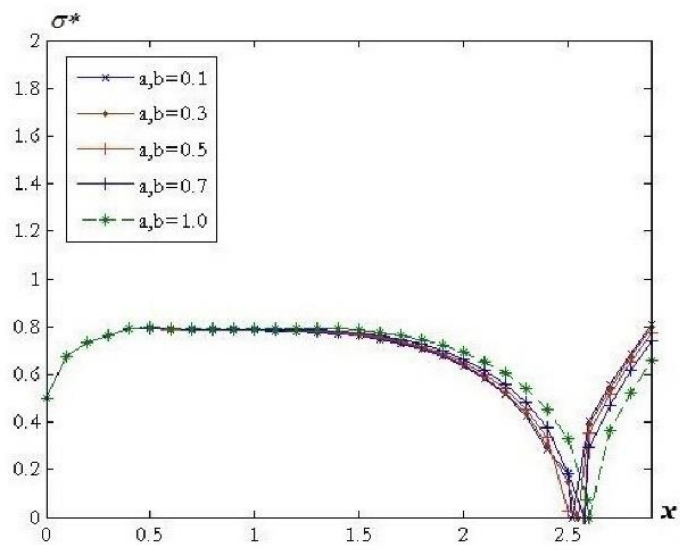

Figure 3: Stable and unstable domains of MHD gravitating jet penetrated by oblique varying magnetic field for $(M, N)=(0.1,0.1)$ with $\sigma^{*}=\sigma / \sqrt{T / \rho R_{o}^{3}}, U=0.5$ and $a=\alpha, B=\beta$.

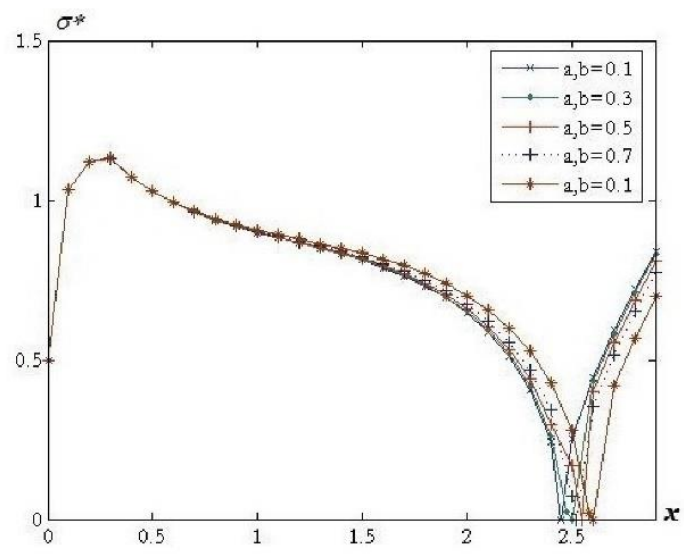

Figure 4: Stable and unstable domains of MHD gravitating jet penetrated by oblique varying magnetic field for $(M, N)=(0.1,0.5)$ with $\sigma^{*}=\sigma / \sqrt{T / \rho R_{o}^{3}}, U=0.5$ and $a=\alpha, B=\beta$.

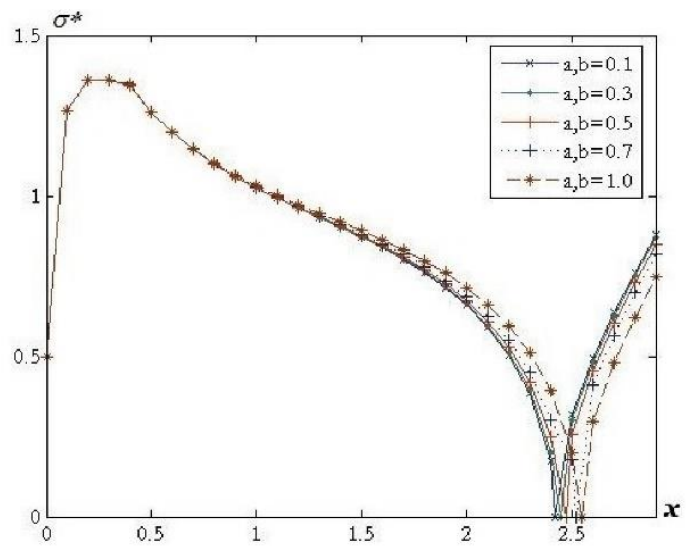

Figure 5: Stable and unstable domains of MHD gravitating jet penetrated by oblique varying magnetic ficld for $(M, N)=(0.1,1.0)$ with $\sigma^{*}=\sigma / \sqrt{T / \rho R^{3}}, U=0.5$ and $a=\alpha, B=\beta$. 


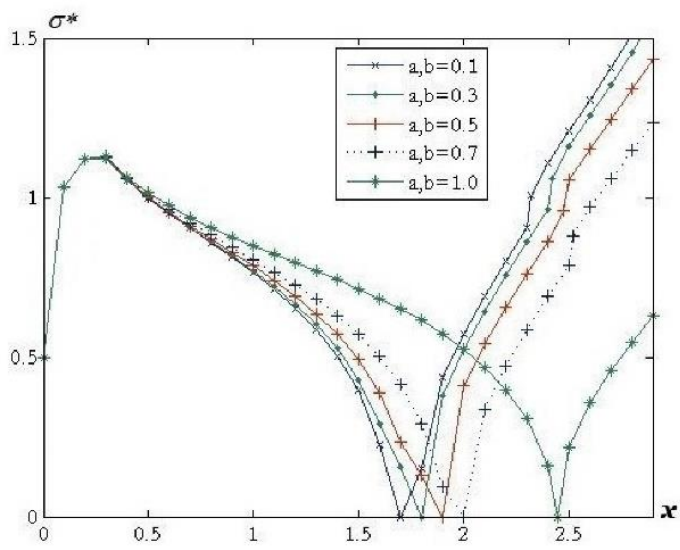

Figure 6: Stable and unstable domains of MHD gravitating jet penctrated by oblique varying magnetic field for $(M, N)=(0.5,0.5)$ with $\sigma^{*}=\sigma / \sqrt{T / \rho R_{o}^{3}}, U=0.5$ and $a=\alpha, B=\beta$.

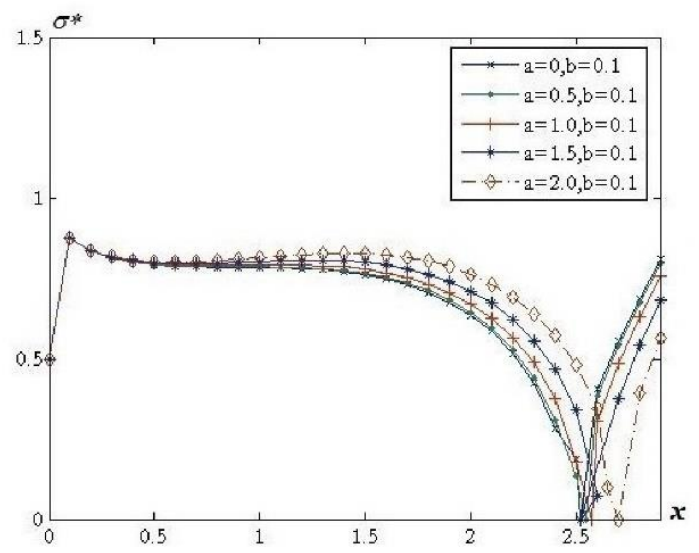

Figure 7: Stable and unstable domains of MHD gravitating jet penetrated by oblique varying magnetic field for $(M, N)=(0.1,0.1)$ with $\sigma^{*}=\sigma / \sqrt{T / \rho R_{o}^{3}}, U=0.5$ and $a=\alpha, B=\beta$.

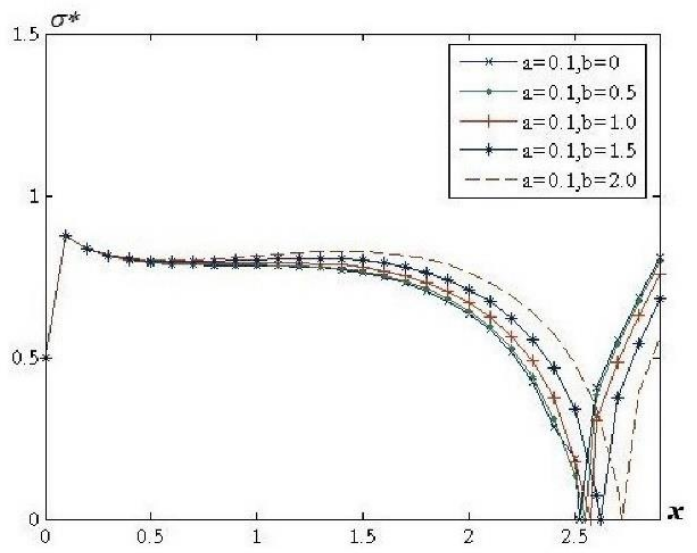

Figure 8: Stable and unstable domains of MHD gravitating jet penctrated by oblique varying magnetic field for $(M, N)=(0.1,0.1)$ with $\sigma^{*}=\sigma / \sqrt{T / \rho R_{o}^{3}}, U=0.5$ and $a=\alpha, B=\beta$. 


\section{REFERENCES}

[1] Abramowitz, Milton; Stegun, Irene A., Handbook of Mathematical Functions with Formulas, Graphs, and Mathematical Tables, Dover pub., New York, USA (1972).

[2] A. A. Hasan, Electrogravitational stability of streaming compound jets, Int J. Biomathematics, 9(3), 2016, 1-13.

[3] A. E. Radwan, "Effect of magnetic fields on the capillary instability of an annular liquid jet," Journal of Magnetism and Magnetic Materials, vol. 72, no. 2, pp. 219-232, 1988.

[4] A. E. Radwan, A. A. Hasan, and A. Y. Sayed, "Electrohydrodynamic Instability of Periodic Streaming Jet", Int. J. of Appl. Math and Mech. 6 (9): 90-110, 2010.

[5] A. E. Radwan, Hydromagnetic Stability of a Streaming Annular Liquid Jet Having a Solid Rod as a Mantle Under Longitudinal Magnetic Fields, Simon Stevin. 64, 1990, 365 .

[6] A. E. Radwan, Instability of a Hollow Jet with Effects of Surface Tension and Fluid Inertia, J. Phys. Soc. Japan, $58,1989,1225$

[7] A.M. Al-Khateeb, N.M. Laham, Magnetic field effects on the Rayleigh-Taylor instability of a stratified fluid plasma in an inhomogeneous plasma, The Arabian Journal for Science and Engineering 27 (2002) 75-83.

[8] A.M. Al-Khateeb, N.M. Laham, Stability criteria of Rayleigh - Taylor modes in magnetized plasmas, Contrib. Plasma Phys. 43 (2003) 25-32.

[9] A. Hasan, R. Abdelkhalek, Magnetogravitodynamic stability of streaming fluid cylinder under the effect of capillary force, Boundary Value Problems, 2013(48)1

[10] B. K. Shivamoggi, Rayleigh-Taylor instability of superposed barotropic fluids, Journal of Applied Mathematics and Physics 63 (2012) 521-527.

[11] Chandrasekhar, S. and Fermi E. Astrophys. J. 118 (1953) 116.

[12] M. G. Cabezas, M. A. Herrada, and José M. Montanero, Stability of a jet moving in a rectangular microchannel, American Physical Society, vol. 100, iss. 5 (2019), https://doi.org/10.1103/PhysRevE.100.053104

[13] Chandrasekhar, S. Proc. Roy. Soc. London 210 (1954) 427.

[14] Dhanesh Ayyappan, Ajith Kumar, Aravind Vaidyanathan), and Nandakumar Study on instability of circular liquid jets at subcritical to supercritical conditions using dynamic mode decomposition, Physics of Fluids 32, 014107 (2020); https://doi.org/10.1063/1.5129240 
[15] M.Rubio, A.Rubio, M.G.Cabezasa, A.Herradab, A.M.Gadn-Calvo and J.M.Montanero, Transonic flow focusing: stability analysis and jet diameter. International Journal of Multiphase Flow, 142, (2021), https://doi.org/10.1016/j.ijmultiphaseflow.2021.103720

[16] Chandrasekhar, S. "Hydrodynamic and Hydromagnetic stability" Dover pub., New York, USA (1981).

[17] Drazin, P. G. and Reid, W.H. "Hydrodynamic stability" Cambridge Univ. Press, London (1980)

[18] Galal M. Moatimid, Doaa R. Mostaphaa, Nonlinear electrohydrodynamic instability through two jets of an Oldroydian viscoelastic fluids with a porous medium under the influence of electric field, AIP Advances 9, 055302 (2019)

[19] Hasan, AA, Electrodynamic stability of two selfgravitating streaming fluids interface, International Journal of Electromagnetics and Mechanics, 53(4), 2017, pp 517-527

[20] Hasan, AA, Electrogravitational stability of oscillating streaming dielectric compound jets ambient with a transverse varying electric field, Boundary Value Problems,2011(31),1.

[21] Hasan, AA, Electrogravitational stability of oscillating streaming fluid cylinder. J Appl Mech ASME. 79(2),(2012)1.

[22] Hasan, AA, Hydromagnetic instability of streaming jet pervaded internally by varying transverse magnetic field. Mathematical Problems in Engineering, (2012)1.

[23] H. K. Moffat, On fluid flow induced by a rotating magnetic field, J. Fluid Mech. 22 (1965) 521-528.

[24] I. Grants, G. Gerebeth, Stability of axially symmetric flow driven by a rotating magnetic field in a cylindrical cavity, J. Fluid Mech. 431 (2001) 407-426. [45] M. Bologna, B. Tellini, F. Giraldi, Plasma in a Rotating Magnetic Field, Plasma Science, IEEE Transactions on 36 (2008) 140-145.

[25] K. Nagaoka, A. Okamoto, S. Yoshimura, M. Kono, M. Y. Tanaka, Spontaneous Formation of a Plasma Hole in a Rotating Magnetized Plasma: A Giant Burgers Vortex in a Compressible Fluid, Phys. Rev. Lett. 89 (2002) 075001.

[26] M. Kono, M. Tanaka, Detection of a Flow Induced Magnetic Field Eigenmode in the Riga Dynamo Facility, Phys. Rev. Lett. 84 (2000) 4369-4372.

[27] M. P. Volz and K. Mazuruk, An experimental study of the influence of a rotating magnetic field on Rayleigh-Bénard convection, J. Fluid Mech. 444 (2001) 79-98.

[28] Ogansion, R. J. Astron 33 (1956) 928.

[29] Lin, C. L. "The theory of Hydrodynamic stability" Cambridge Univ. Press, London, UK. (1976).

[30] Rayleigh J. W. "The Theory of Sound" Vols. I \& II. Dover pub., New York, USA (1945).

[31] Radwan, A. and Debnath, L., Comput. Math. Appl. UK 31 (1996) 61. 
[32] Radwan, A. and Aly, F., IL Nuovo Cimento 113 (1998)1.

[33] Radwan, A and Sileem, S., Chaos, Solitoms \& Fractels 12 (2001) 1729.

[34] Radwan, A. and Hashem, H., Appl. Maths. \& Comput. 147 (2004) 191.

[35] Radwan, A. and Hasan, A. Appl. Math. Model. UK 33 (2009) 4.

[36] Radwan, A. and Hasan, A. , (IAENG) Int. J. Appl. Maths, 38(3)(2008) 113

[37] R. J. Goldston, P. H. Rutherford, Introduction to Plasma Physics Institute of Physics, London, 1997.

[38] Samia S.Elazab, Alfisal A. Hasan and Ahmed Y. Sayed, "Capillary Magnetogravitational Instability of Fluid Cylinder with Varying Magnetic Field", Fifth Saudi Science Conference, Vol. P05 (2012), p240.

[39] X. Ribeyre, V. T. Tikhonchuk and S. Bouquet, Compressible Rayleigh-Taylor instabilities in supernova remnants, Phys. Fluids 6 (2004) 4661-4670. 